\title{
COMMENTARY
}

\section{Femtosecond-laser nanolithography for photonic applications}

\author{
Hiroaki Nishiyama \\ Division of Materials and Manufacturing Science, Graduate School of Engineering, Osaka \\ University, 2-1 Yamada-oka, Suita, Osaka, Japan \\ hiroaki@mapse.eng.osaka-u.ac.jp
}

In 1997, Kawata et al. reported a surprising experimental result that full three-dimensional (3D) microstructures had been fabricated by photopolymerization [1]. Four year later, the world's smallest 3-D bulls were created by the same research group [2]. The bulls were based on polymeric materials and were approximately $10 \mu \mathrm{m}$ in size, similar to that of human red blood cells. The spatial resolution of their fabrication technique was as high as approximately $100 \mathrm{~nm}$, which is beyond the diffraction limit. Recently, the much higher resolution of sub-30 nm was achieved using 3-D bridge structures [3].

These 3-D structures were created using femtosecond laser direct writing and subsequent development treatment. As illustrated in Fig. 1, when femtosecond laser pulses are focused tightly into transparent materials, photochemical reactions occur only near the focal volume via nonlinear optical processes such as multiphoton absorption (MPA), because the rate of MPA depends strongly on light intensity. For instance, the two-photon absorption rate is proportional to the square of the light intensity. Because of this characteristic, the MPA technique enables us to expose the internal region directly and to create complex polymeric micro/nano-structures by translating a focal spot inside the resin. The strong dependence of MPA also allows for confinement of laser-modified areas to sub-micrometer volume.

Although semiconductor-manufacturing technology is well established for the production of arbitrary patterns with ultrahigh spatial resolution, it is rather difficult to create complicated 3-D structures because of their planar nature. For example, we are unable to fabricate microstructures on 3-D (non-flat) substrates. In fact, all electronic circuits are planar (or multilayer) structures in microelectronics field. Focused-ion-beam chemical-vapor-deposition

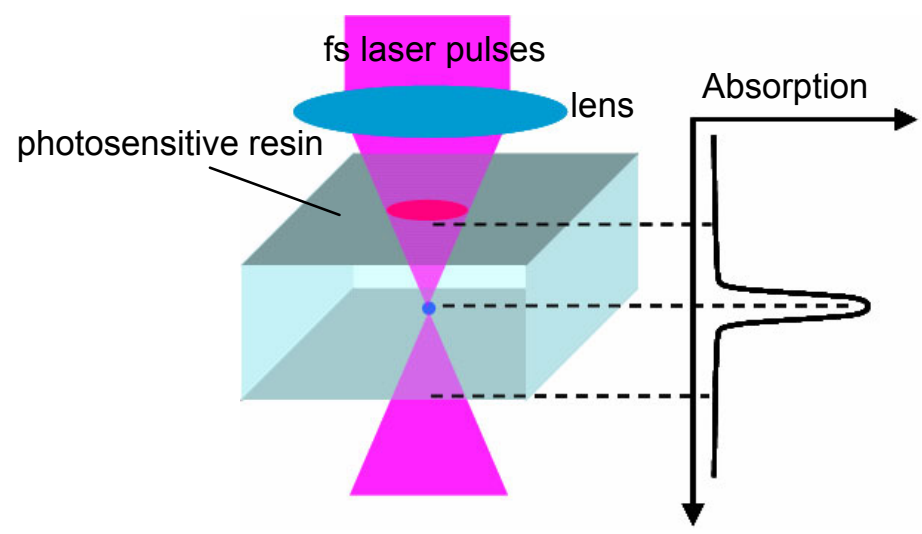

Fig. 1. Schematic illustration of multiphoton absorption induced using femtosecond laser pulses. 
(CVD) is a promising technique for 3-D microstructures [4]. However, usable materials are considerably limited, and large vacuum chambers are required. In contrast, the MPA technique can use various photosensitive materials including SCR-500, SU-8, and organicinorganic hybrid materials. In addition, inexpensive laser sources for MPA are commercially available.

Photonic devices are one of attractive applications for the MPA technique. To date, several research groups have demonstrated the fabrication of polymeric 3-D photonic crystals with sub-micrometer periods for generation of stop-band-gap at the optical communication wavelength. Although only photosensitive polymers are usable for the MPA technique, it is desirable to incorporate other materials such as metals and semiconductors into 3-D structures for many applications. Therefore, in the past several years, combination of the MPA technique and other methods has been studied intensively. Mizeikis et al. created metallodielectric hybrid materials through chemical modification of 3-D structures formed by the MPA technique for metamaterial applications [5]. Several groups demonstrated the fabrication of Si 3-D photonic crystals with line defect-waveguides through combination with CVD technology $[6,7]$.

We developed a technique that forms 3-D surfaces of inorganic optical materials using a combination of the MPA technique and commonplace semiconductor technology [8,9]. This approach was named as femtosecond-laser lithography-assisted micromachining (FLAM). In this method, we transfer 3-D resist structures created by the MPA technique to an underlying layer of inorganic materials with reactive plasma. Surface profiles of micro-photonic devices including diffractive elements and plasmon devices strongly affect their optical properties. If semiconductor technology is applicable to non-flat substrates such as convex lenses, various 3-D surfaces with high quality will be obtained, leading to the formation of highly functional photonic devices. The most serious hindrance to microfabrication onto non-flat substrates is deformation of the resist. When the resist is coated upon non-flat substrates, the resist thickness varies from area to area, mainly due to surface tension. Because the photon energy from lithography light sources is absorbed from the resist surface via one-photon process, it is difficult to form precise microstructures, even with highly accurate laser control. To overcome this problem, we used direct internal exposure in the MPA technique.

Figure 2 shows an $\mathrm{SEM}$ image of $\mathrm{SiO}_{2}$-based refractive-diffractive hybrid microlenses fabricated by FLAM. The diameter and curvature radius of a microlens are, respectively, 240 $\mu \mathrm{m}$ and $380 \mu \mathrm{m}$. Fresnel zone plate patterns were written directly inside a negative-tone resist
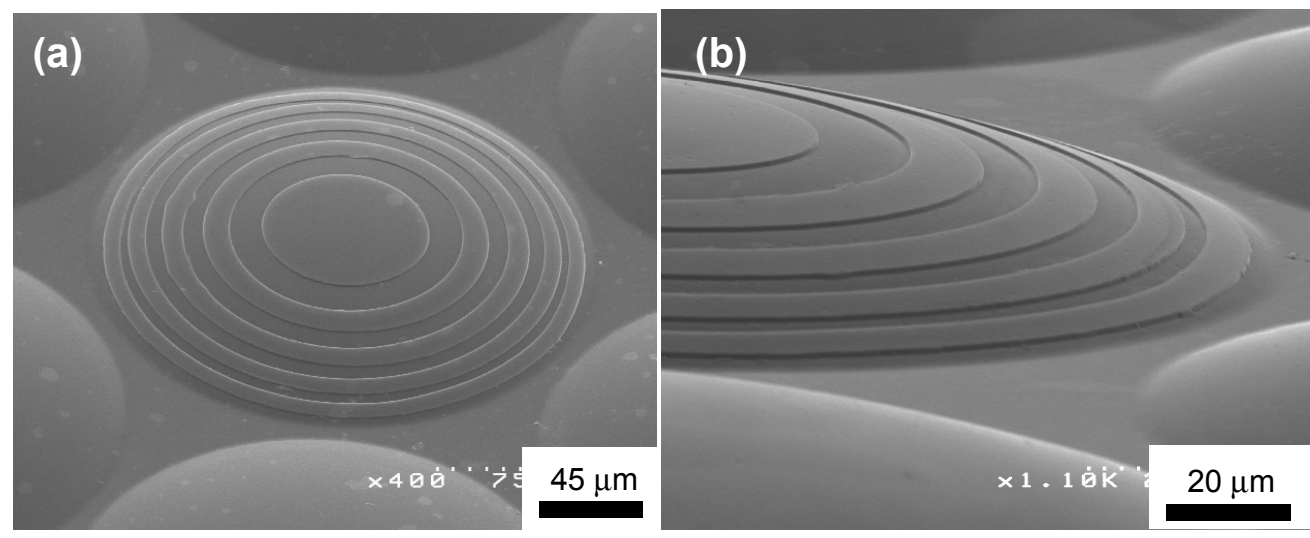

Fig. 2. SEM images. (a) overview and (b) enlarged view of an $\mathrm{SiO}_{2}$-based hybrid lens. Reprinted with permission from Optics Express. Copyright 2008, Optical Society of America [8]. 
coated on the convex microlenses. By post-exposure-baking, the cross-linking reaction of monomers was induced in exposed regions. Unexposed regions were washed away by development treatment. We obtained hybrid lenses by transferring the zone plate patterns to the underlying convex lenses by $\mathrm{CHF}_{3}$ plasma. From Fig. 2, it is readily apparent that welldefined structures with smooth surfaces were produced on lenses in spite of deformation of the resist.

The wavelength-dispersion properties of diffractive lenses are opposite to those of refractive counterparts. Therefore, the hybridization of both types of lenses enables us to compensate for chromatic aberration and control focal lengths. Here, focal lengths of the hybrid lenses were designed to be shorter than those of original convex lenses. When 632.8$\mathrm{nm}$-wavelength He-Ne laser light was normally coupled to the hybrid lens, the primary focal length was found to be $614 \mu \mathrm{m}$. Because of the hybridization, the focal length changed by 216 $\mu \mathrm{m}$. This shift agreed well with the theoretical value of $213 \mu \mathrm{m}$. Using FLAM, we were able to fabricate precise microstructures even across a step of $85 \mu \mathrm{m}$ height. Although femtosecond laser pulses were focused by a low-numerical-aperture lens in our experiments, spatial resolutions of sub-diffraction limits were also obtained. Furthermore, FLAM is applicable to various plasma-etchable materials including $\mathrm{Si}, \mathrm{SiO}_{2}$, and GaN. FLAM is expected to be useful for the realization of functional photonic devices with finer and more complex 3-D surfaces.

Three-dimensional micro-electro-mechanical systems (MEMS) and metamaterials are attractive applications for use of the MPA technique. Such applications strongly require 3-D microfabrication techniques with inorganic materials including metals and semiconductors. To address those needs, over the next few years, hybridization of the MPA technique with other techniques must be explored more extensively. From the perspective of quality of structures, dry processes such as CVD technology might be more promising for the hybridization than solution-phase ones. Although the MPA technique is a powerful tool for 3D microfabrication, the throughput remains insufficient for mass production. Therefore, 3-D moldings and replication approaches [10] such as nanoimprint lithography will be of growing importance. For novel functional devices in various fields such as photonics, microelectronics, MEMS devices, and microfluidics, the MPA technique is expected to play an essential role.

\section{Acknowledgments}

H. N. deeply appreciates Prof. Y. Hirata and Ms. M. Mizoshiri (Osaka Univ.) for helpful discussions.

\section{References}

[1] S. Maruo, O. Nakamura, and S. Kawata, "Three-dimensional microfabrication with two-photon-absorbed photopolymerization," Opt. Lett. 22, 132-134 (1997) [doi:10.1364/OL.22.000132].

[2] S. Kawata, H. B. Sun, T. Tanaka, and K. Takada, "Finer features for functional microdevices," Nature 412, 697-698 (2001) [doi:10.1038/35089130].

[3] S. Juodkazis, V. Mizeikis, K. K. Seet, M. Miwa, and H. Misawa, "Two-photon lithography of nanorods in SU-8 photoresist," Nanotechnology 16, 846-849 (2005) [doi:10.1088/0957-4484/16/6/039].

[4] S. Matsui, T. Kaito, J. Fujita, M. Komuro, K. Kanda, and Y. Haruyama, "Threedimensional nanostructure fabrication by focused-ion-beam chemical vapor deposition," J. Vac. Sci. Technol. B 18, 3181-3184 (2000) [doi:10.1116/1.1319689].

[5] V. Mizeikis, S. Juodkazis, R. Tarozaite, J. Juodkazyte, K. Juodkazis, and H. Misawa, "Fabrication and properties of metalo-dielectric photonic crystal structures for 
infrared spectral region," Opt. Express 15, 8454-8464 (2007)

[doi:10.1364/OE.15.008454].

[6] N. Tétreault, G. von Freymann, M. Deubel, M. Hermatschweiler, F. Perez-Willard, S. John, M. Wegener, and G. A. Ozin, "New route to three-dimensional photonic bandgap materials: Silicon double inversion of polymer templates," Adv. Mater. 18, 457-460 (2006) [doi:10.1002/adma.200501674].

[7] S. A. Rinne, F. García-Santamaria, and P. V. Braun, "Embedded cavities and waveguides in three-dimensional silicon photonic crystals," Nature Photon. 2, 52-56 (2008) [doi:10.1038/nphoton.2007. 252].

[8] H. Nishiyama, M. Mizoshiri, T. Kawahara, J. Nishii, and Y. Hirata, " $\mathrm{SiO}_{2}$-based nonplanar structures fabricated using femtosecond laser lithography," Opt. Express 16, 17288-17294 (2008) [doi:10.1364/OE.16.017288].

[9] M. Mizoshiri, H. Nishiyama, T. Kawahara, J. Nishii, and Y. Hirata, " $\mathrm{SiO}_{2}$-based hybrid diffractive-refractive lenses fabricated by femtosecond laser-assisted micromachining," Appl. Phys. Express 1, 127001 (2008) [doi:10.1143/APEX.1.127001].

[10] C. N. LaFratta, T. Baldacchini, R. A. Farrer, J. T. Fourkas, M. C. Teich, B. E. A. Saleh, and M. J. Naughton, "Replication of two-photon-polymerized structures with extremely high aspect ratios and large overhangs," J. Phys. Chem. B 108, 1125611258 (2004) [doi:10.1021/jp048525r]. 